\title{
Towards fabrication and application of polymer based photonics networks and sensors
}

Maik Rahlves, Maher Rezem, Axel Günther, Christian Kelb, Muhammad Khan, et al.

Maik Rahlves, Maher Rezem, Axel Günther, Christian Kelb, Muhammad Khan, Eduard Reithmeier, Bernhard Roth, "Towards fabrication and application of polymer based photonics networks and sensors," Proc. SPIE 10545, MOEMS and Miniaturized Systems XVII, 1054502 (22 February 2018); doi: $10.1117 / 12.2300311$

SPIE. Event: SPIE OPTO, 2018, San Francisco, California, United States 


\title{
Towards fabrication and application of polymer based photonics networks and sensors \\ Maik Rahlves*a, Maher Rezemª, Axel Günther ${ }^{\mathrm{a}}$, Christian Kelb ${ }^{\mathrm{a}}$, Muhammad Khan ${ }^{\mathrm{a}}$, Eduard Reithmeier ${ }^{\mathrm{b}}$, Bernhard Roth ${ }^{\mathrm{a}}$

\author{
${ }^{a}$ Hannover Centre for Optical Technologies, Leibniz Universität Hannover, Nienburger Strasse 17,

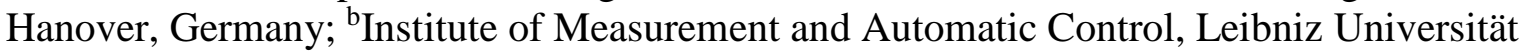 \\ Hannover, Nienburger Strasse 17, Hanover, Germany
}

\begin{abstract}
Highly-functional photonic sensor networks integrated in thin polymer foils offer great potential for versatile applications in the life sciences, medicine, environmental analytics or production technology. For their realization, suitable low-cost and high-throughput production techniques need to be developed. Here, we describe work towards this goal, i.e. the fabrication of multimode polymer waveguides through a combination of thermal imprint and doctor blading. For imprint master stamp fabrication, a combined Bosch and $\mathrm{O}_{2}$ plasma etching process in silicon is utilized. We also demonstrate stamp fabrication by an additive manufacturing method, i.e. by employing maskless UV lithography, to enhance the flexibility and cost-effectiveness of our approach. We, thus, realize various all-polymer waveguide arrays, beam splitters, and grating couplers which serve as basic elements to create more complex photonic circuits. We also demonstrate polymer based transmission lines comprising semiconductor as well as organic light sources and detectors. We discuss both the integration of semiconductor light sources and detectors such as verticalcavity surface-emitting lasers (VCSEL) and photo detectors as well as organic light emitting diodes (OLEDs) and organic photo detectors. In first applications, we combine these elements to create sensor arrays for measuring temperature, strain or refractive index. We show results of various sensor types utilizing different measurement principles implemented in laboratory environments so far. For example, a waveguide array containing a linear discontinuity which serves as elongation zone for displacement, strain or tilt measurement by detecting the intensity variation of the transmitted light propagating inside the structure is presented. In future, we plan to create more powerful sensor photonics networks for reliable and robust applications in real life, e.g. for point-of-care testing or production monitoring.
\end{abstract}

Keywords: Hot embossing, optical micro-structures, polymer waveguide-arrays, optical interconnects, polymer optics, integrated photonics

\section{INTRODUCTION}

The advances in polymer-based photonics achieved during the last decade open the route towards more versatile application scenarios in distributed sensing and analytics based on artificial skin-like sensor networks, see Figure 1 for a possible implementation. Compared to their glass or silicon-based counterparts polymer photonic devices feature several compelling advantages such as cost and resource efficiency, biocompatibility and flexibility [1,2]. Also, on the material side, the optical properties of the underlying polymer species, for example, the viscosity and the refractive index can be adjusted according to the application requirements, by suitable chemical additives [3]. In addition, their insensitivity with regard to electromagnetic noise (in common with glass-based optics) and their promising multiplexing capabilities exceeding those of conventional fiber optics, for example, render them in many cases superior to electronic or microelectronic systems [1]. With regard to fabrication, the field can only partially benefit from silicon based manufacturing techniques which are already established at the industrial scale as these are usually cost and time intensive and not sufficiently adaptable. In contrast to these techniques, the field focuses more on high-yield processing and assembly ultimately aiming at roll-to-roll (R2R) and roll-to-plate (R2P) technology or on employing additive manufacturing such as laser direct writing or two-photon polymerization, among others. However, to realize large-scale functionalized polymer foils with integrated optical sensors, the production techniques developed so far for individual polymer-based optical elements, e.g. micro-optics and basic wave guiding structures, need to be further developed and extended to meet 
demands dictated by mass-market applications and flexible measurement scenarios. Consequently, the production technologies needed shall not only be low-cost and high-throughput capable but incorporate all relevant process steps to integrate the required light sources, detectors and sensing structures into the polymer foil substrate. As reliability and robustness as well as sensor functionality and performance are among the main challenges to be addressed in this context, it is of paramount importance that the structures fabricated in a potentially multi-step process are not affected by successive steps.

Thus, the successful realization of process chains to generate entire polymer-optical sensor networks provided holds great promise to substantially advance application of the systems in fields not accessible so far with this technology. For example, in point-of-care testing (POCT) simple and yet reliable sensor devices are desirable to continuously monitor patient heath conditions or healing processes, both by trained and also untrained personnel. Also, wearable and integrated polymer-based optics lend themselves for these applications as they fulfil all requirements with regard to cost, flexibility, sensitivity, and eventually biocompatibility [4]. Such systems could in future not only be established for remote medical diagnostics or disposable lab-on-chip optofluidics, e.g. in resource-limited regions of the world, but also for on-demand monitoring of environmental pollution or process parameters in production environments which are difficult to access. Ultimately, various in-line or real-time measurement scenarios in bioreactors, food production, security, ambient assisted living or structural health monitoring (SHM) appear feasible.

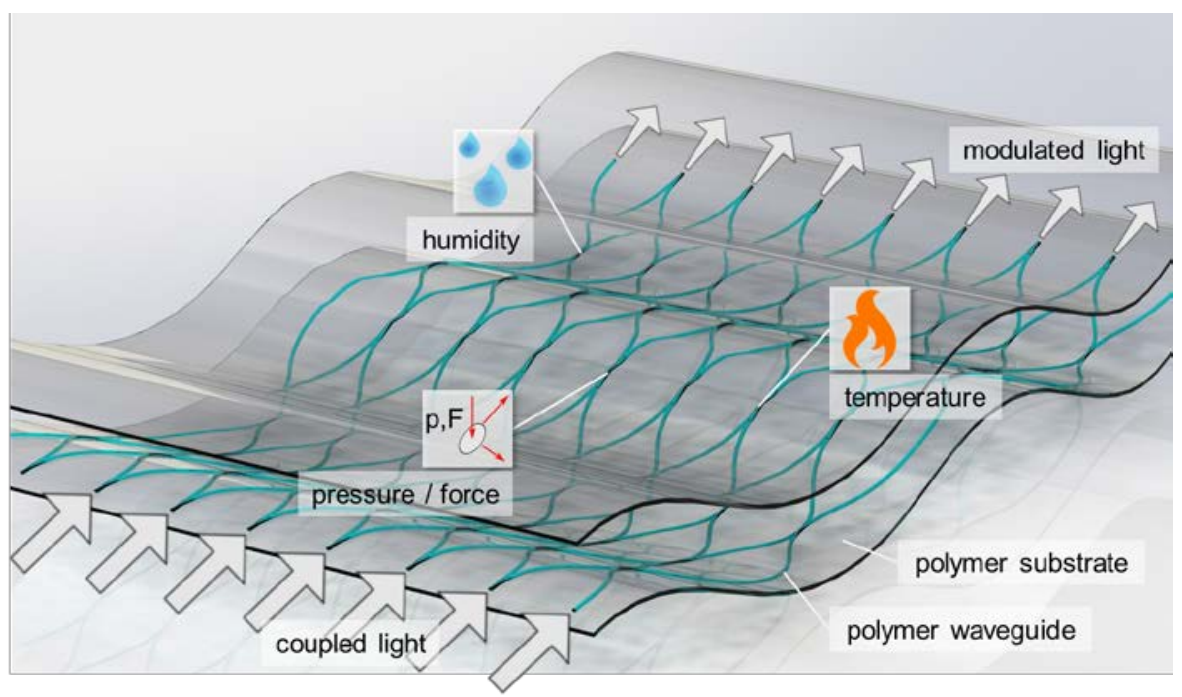

Figure 1. Visionary example for a planar polymer-optical sensor network for 2D distributed measurement of quantities such as pressure/force, humidity and temperature. The quantities to be detected are converted into optical signals thereby omitting electronic components as far as possible. In future, such sensors networks could be manufactured in an R2R process, for example, featuring high multiplexing capabilities and spatial resolution.

In this work, we present technologies developed along these lines which enable the production of basic sub-systems of such polymer sensor networks, for example embedded polymer waveguide structures as well as optical couplers and beam splitters. Based on the results achieved so far we demonstrate possibilities for combination into integrated optical transmission lines and sensor units and discuss the sensitivities obtained, see also [5-8]. We first discuss fabrication of low-loss planar multimode optical waveguides based on hot embossing, doctor blading and UV curing. Then, fabrication of low-loss interconnects and coupling structures are presented, which are required for the integration of optical sources, detectors and fibers. Finally, we exemplarily describe the performance of a polymer-optical strain sensor system realized by using these techniques and discuss the system performance with regard to the applications envisioned in future.

\section{ALL-POLYMER MULTIMODE OPTICAL WAVEGUIDES}

For the fabrication of multimode optical waveguides we employ two technologies. One relies on hot embossing of thermoplastics followed by doctor blading, the second is based on maskless projection photolithography in photoresist. Both processes are capable of manufacturing multimode waveguide structures and beam splitters elements. Also, we utilize low-cost commercially available materials exhibiting low optical losses which are suited for mass production in future. 


\subsection{Fabrication Methods and Polymer Materials}

a) Hot embossing and doctor blading of polymer-optical waveguides

The waveguides required for polymer photonic systems are usually manufactured either by direct structuring of polymers deposited on silicon or polymer substrates or by hot embossing in combination with spin-coating or doctor blading. Usually these processes require relatively expensive master tools, e.g. photomasks or hot-embossing stamps made from silicon. However, it was shown that they enable the production of both multimode and single mode optical waveguides with low losses.

The fabrication of multimode optical waveguides in our work consists of several steps: hot embossing of the cladding structure and doctor blading of the waveguide core followed by an UV-curing step. The developed process is sketched in Figure 2, left panel.

a)

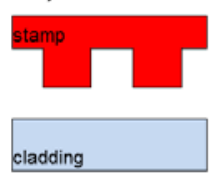

b)

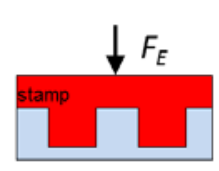

e)

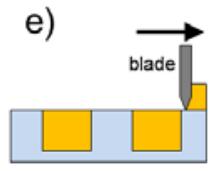

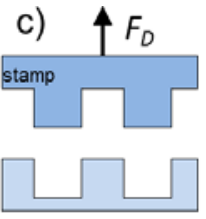

f)

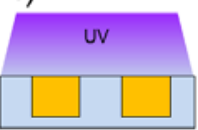

d)

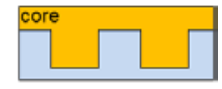

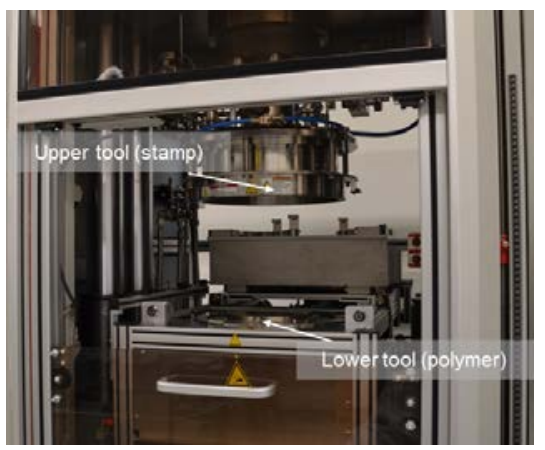

Figure 2. Left panel: Overview of the developed optical waveguide fabrication process chain: (a) heating of stamp and cladding material, (b) hot embossing of cladding structure, (c) cooling and demolding step, (d) deposition of the core material through spin-coating, (e) doctor blading step, and (f) UV-curing of the core material (from [9]). Right panel: photograph of the central part of the hot embossing machine used in this work.

As substrate material representing the cladding of the waveguide structures manufactured we use the thermoplastic polymethylmethacrylate (PMMA) which is particularly suited for hot embossing. The hot embossing process relies on transferring the structures of a master stamp onto a polymer substrate, thus, creating a negative replica of the stamp. Our experimental setup utilizes a commercial hot embossing machine (HEX03, Jenoptik Mikrotechnik GmbH), see Figure 2, right panel. The machine works with a plate-to-plate configuration with typical embossing temperatures of up to $300^{\circ} \mathrm{C}$ and embossing forces of up to $300 \mathrm{kN}$. Various master stamps were used for replication. In the examples discussed here, a silicon wafer (Micromotive $\mathrm{GmbH}$ ) was employed, see Figure 3. The wafer is patterned through photolithography and deep reactive-ion etching and contains waveguide and coupling structures of various geometries.

Ideally, in order to minimize scattering losses of the optical waveguides manufactured, the stamp surface roughness should be on the order of a few $\mathrm{nm}$. As this roughness is transferred to the negative replica during the hot embossing process, this directly affects the surface scattering properties of the waveguide core created in the subsequent step. Thus, the processing of the silicon wafer with plasma etching in its last fabrication step is required to ensure good surface quality. In our case, this was verified with a laser scanning microscope (VK X100, Keyence, Japan) where we obtained an average surface roughness $R_{a}$ of the cladding structure of $6 \mathrm{~nm}$. As displayed in Figure 3, the stamp contains straight and bent rib-structures with height and width of $25 \mu \mathrm{m}$, respectively, which after the hot embossing step lead to embedded structures to be filled with core material.

Initially, for hot embossing, a 500 $\mu \mathrm{m}$ thin PMMA substrate and the silicon stamp were heated to a temperature of $140^{\circ} \mathrm{C}$ prior to the forming step. At this temperature, the PMMA reaches the so-called rubbery state $[10,11]$ and pattern replication can start. The subsequent embossing step, starts by applying an embossing force of $4 \mathrm{kN}$ between the stamp and the substrate which is usually maintained for 2 minutes. During this phase, the stamp structures are transferred on the PMMA surface. The force continues to be maintained in the cooling step following the patterning, where the stamp and the thermoplastic cooled down to temperatures of approximately $50^{\circ} \mathrm{C}$. This is when the final demolding step starts: the embossing force is slowly released and the stamp and PMMA are separated by hand. Following the hot embossing 
step, the doctor blading step is initiated. Here, a liquid uncured waveguide material is deposited on the PMMA cladding by spin-coating and the superfluous material is removed by using a razor blade, see Figure 2. Although various other processes can be used to apply the core material, the one employed here is particularly simple and can be transferred to a future R2R process. Also, it leads to very thin residual layers. The final manufacturing step consists of a UV-curing process to harden the core material. For the main materials investigated in our work, i.e., the low-cost optical adhesives NOA68 (Norland, USA), OG198-54 (Epotek, USA), and OG142 (Epotek, USA), a UV-lamp emitting at a wavelength of $365 \mathrm{~nm}$ was used for the curing step. Figure 3 (right panel) shows examples of hot embossed cladding (top) and core waveguide structures (bottom). In addition, using thermal bonding, multi-laxer structures containing embedded and surface waveguide layers were realized, see [12]. Such structures are particularly suited for sensing as the upper waveguide layer is ideal to sense quantities of the environment whereas the embedded waveguide layer could serve as reference. In addition to the master tools presented thus far, we also used various other master tools in our studies, see Figure 4 for examples.
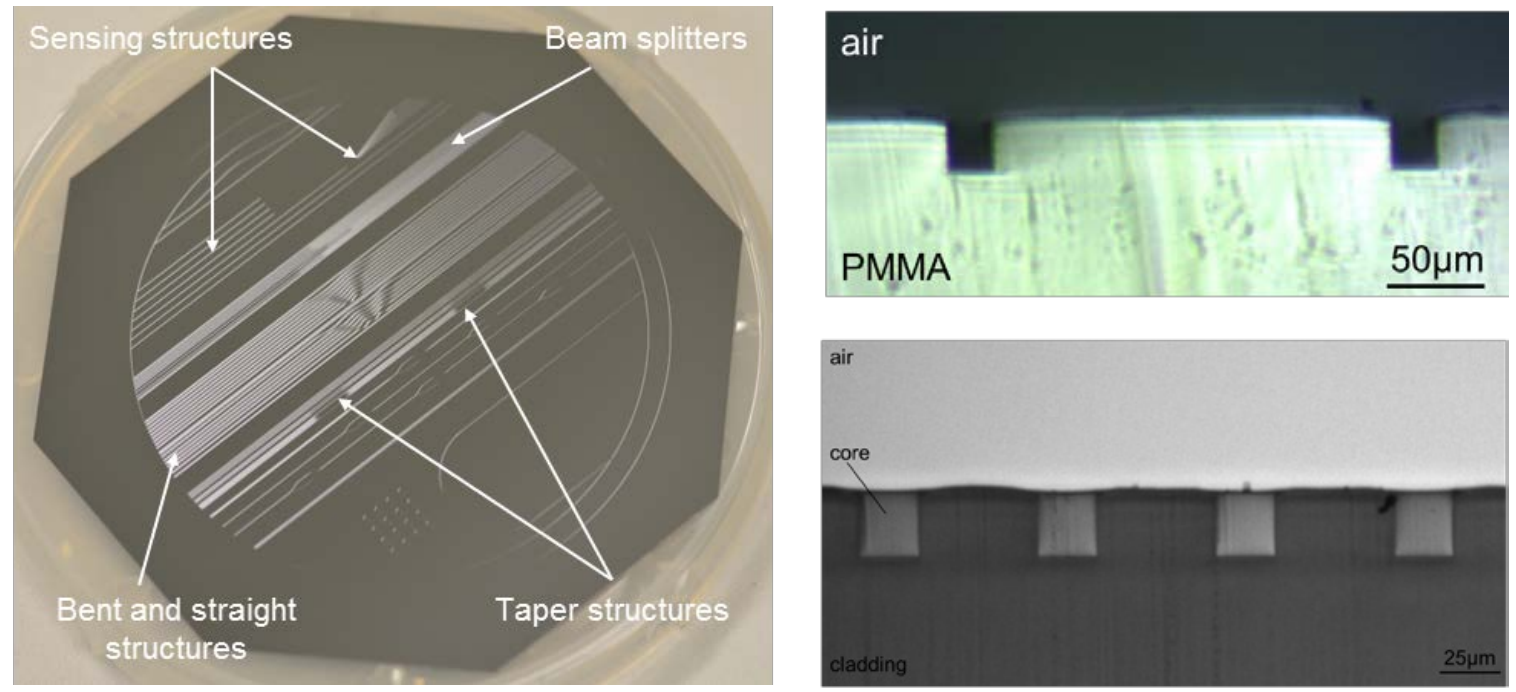

Figure 3. Left: 4-inch silicon stamp used for hot embossing. Right: Trench structures replicated in PMMA (top) and waveguide structures created through the doctor blading and curing step (bottom).
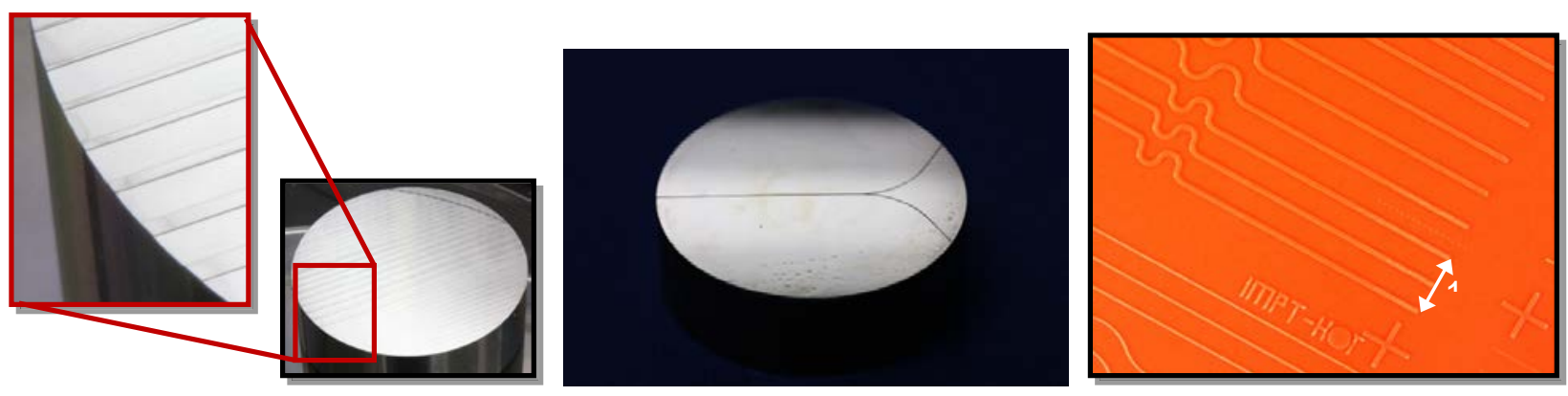

Figure 4. Left and middle: master tools realized by precision milling containing straight rib-structures in aluminum and bent trench structures in tin, respectively. Right: Rib-structures on a silicone embossing stamp, see [13]. 


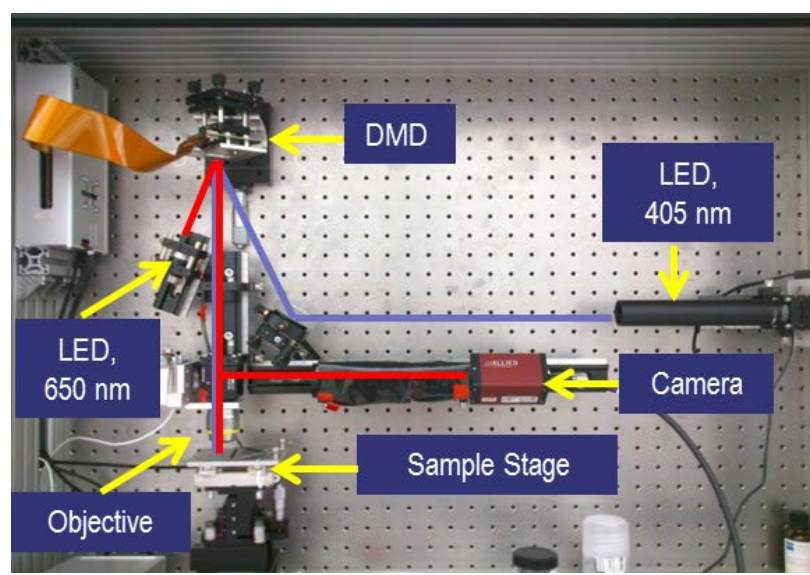

(1) Lithography

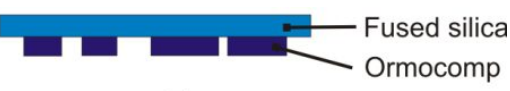

$\$ \$ \downarrow^{\mathrm{F}}$

(2) Hot embossing

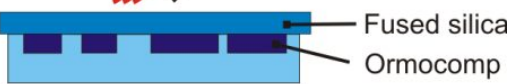

(3) Doctor blading

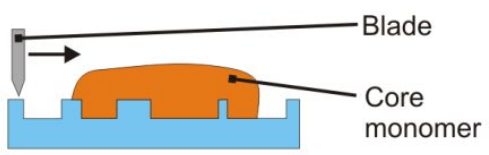

(4) Casting

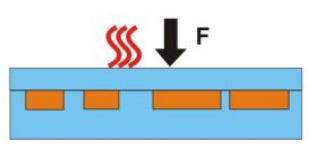

Figure 5. Left: Photograph of the maskless photolithographic setup utilized for waveguide fabrication. Right: Hot embossing process based on master tools produced via maskless lithography (from [16]).

\section{b) Maskless Photolithography}

For the realization of micro-structures such as optical waveguides or diffractive optical elements in a liquid hybrid monomer, we developed a maskless lithographic setup, as illustrated in Fig. 5 (left), see also [14, 15]. The pattern of the desired micro-structure or optical element which is to be transferred into polymer is created by a digital-mirror-device (DMD, Texas Instruments DLP7000) and a personal computer. The DMD serves as spatial light modulator (SLM) and features a pixel pitch of $13.68 \mu \mathrm{m}$ and a total pixel number of $1024 \times 768$. The setup contains two light emitting diodes (LED) sources. For the lithographic process, we utilize a high power LED with maximal output power of $0.9 \mathrm{~W}$ and center wavelength at around $405 \mathrm{~nm}$. This wavelength corresponds to the h-line of a mercury vapor lamp. In addition, in order to determine the optimal focal position for processing of the sample, we use an LED at a center wavelength of $650 \mathrm{~nm}$. As typical photoresists and monomers used are commonly not sensitive in this range, thus, the LED at $650 \mathrm{~nm}$ does not alter their material properties. This enables us to visualize the sample while finding the best focal position for photolithography. The LED light sources are controlled via an Arduino microcontroller board integrated into the setup.

We use a standard microscope setup (Carl Zeiss Microscopy) to de-magnify the DMD image and to project its pattern onto the sample material. In more detail, we used a microscope objective (Carl Zeiss EC Epiplan Apochromate) with a numerical aperture (NA) of 0.3 and a magnification of 10. Furthermore, the setup contains an infinity corrected tube lens also from Carl Zeiss. To determine the best focal position for lithography, we record images of the substrate by using a CCD camera (pike F421, AVT), see Figure 5 (left). The best focal position is found automatically by moving the microscope objective in steps of $1 \mu \mathrm{m}$ in an interval of $500 \mu \mathrm{m}$ utilizing a piezoelectric linear stage (SLC-1780-S, Smaract). Thereby, by applying a variance based algorithm, we determine the microscope objective position which leads to the sharpest image of the substrate and adjusted its position accordingly. The microscope field of view is $1.9 \mathrm{~mm}$ x $1.1 \mathrm{~mm}$ and is limited by the microscope setup. This also limits the maximum area which can be structured by the current version of our lithographic setup in a single exposure process. To create larger structures, an $\mathrm{x}$-y-lateral linear translation stage (two stages SLC-1780-S with orthogonally orientated axes, Smaract) is incorporated into the setup. This enables multiple exposures at different substrate areas sequentially which allows a maximal area accessible for the lithographic process of $5 \times 5 \mathrm{~cm}^{2}$.

Initially, fabrication of waveguides or integrated optical elements starts with a lithographic process step to form the optical structures. In this step, a thin layer of Ormocomp (Microresist Technology) hybrid polymer is deposited onto a microscope cover slide by spin-coating at a rotational speed of $4000 \mathrm{rpm}$. To achieve a coating thickness in the few micron range, $5 \mathrm{ml}$ Ormocomp are diluted using Ormothin thinner (Microresist Technology) at a ratio between 1:1 to 1:2 which leads to a reduction of the material viscosity. Subsequently, a prebake of the sample is applied (at $80^{\circ} \mathrm{C}$, for $2 \mathrm{~min}$ ) to vaporize the remaining thinner. For lithography, the cover slide is placed onto the lateral translation stage with the Ormocomp layer facing upwards. Following the autofocus process, the lithographic step is performed by uploading a bitmap image of the desired optical structure onto the DMD device and illuminating the sample for 180 seconds. After exposure, a second bake process step is applied to the cover slide (at $150^{\circ} \mathrm{C}$, for $10 \mathrm{~min}$ ) before the slide is submerged into a developer bath (OrmoDev, Microresist Technology) for $1 \mathrm{~min}$ and finally rinsed with pure water. 
Using the master tool fabricated as describe above, embedded waveguides, beam splitters and Mach-Zehnder interferometric structures are realized in a subsequent hot embossing process step similar to the one in Figure 2, see Figure 5 (right). The cover slide including the Ormocomp structures serve as hot embossing stamp to transfer a negative replica of the structures from lithography into PMMA foil. To ensure that the Ormocomp structures remain on the cover slide during the process, a lower embossing temperature of $130^{\circ} \mathrm{C}$ and a lower force of $2 \mathrm{kN}$ are applied. After hot embossing, the substrate with the replicated grooves are filled with a liquid monomer (390119 UV Supraflex, Jänecke+Schneemann Druckfarben, Germany) to form the waveguide cores. Again, a doctor blading and UV-curing process is applied to the PMMA substrate to ensure optically smooth waveguide surfaces and avoid unfavorable residual layers on top of the PMMA. Examples of structures created in this process are illustrated in Figure 7 and their properties are discussed below.

\subsection{Manufactured Structures and their Characterization}

a) Hot Embossed Waveguides

Typically, the first characterization step of newly manufactured waveguides consists of a visual inspection of the end facets of the waveguide which are prepared using an in-house cutting tool. For this purpose, an image of the respective waveguide cross-section is taken using an optical microscope. Following this step, the refractive index distribution of the core materials is measured based on the refracted near-field method by using a refractive index profiler (Rinck Elektronik $\mathrm{GmbH}$ ). Typical measured refractive indices of the waveguide core materials at a temperature of $20^{\circ} \mathrm{C}$ and a wavelength of $638 \mathrm{~nm}$ are given in Table 1.

Table 1. Measured refractive indices of the waveguide core materials used in our work.

\begin{tabular}{|c|c|}
\hline $\begin{array}{c}\text { Waveguide core } \\
\text { material }\end{array}$ & $\begin{array}{l}\text { Measured refractive index } \\
\left(20^{\circ} \mathrm{C}, 638 \mathrm{~nm}\right)\end{array}$ \\
\hline OG142 & 1.568 \\
\hline OG198-54 & 1.524 \\
\hline NOA68 & 1.525 \\
\hline
\end{tabular}

Furthermore, we determine the propagation losses of the fabricated optical waveguides at two wavelengths, $633 \mathrm{~nm}$ and $850 \mathrm{~nm}$, by employing the established cut-back method [18]. For this purpose, the insertion loss of a specific waveguide at different lengths, starting with the full length initially manufactured, is measured. Using the values obtained and linear regression fitting of the data, we determine the propagation losses of the waveguide in $\mathrm{dB} / \mathrm{cm}$. Light coupling into the investigated structures is achieved by a helium-neon laser (25-LHP-991, Melles Griot) at $633 \mathrm{~nm}$ and a microscope objective which focuses the laser beam at the waveguide input facet. At $850 \mathrm{~nm}$, we use a fiber coupled laser diode (MCLS1-850, Thorlabs). Also, for all measurements, the samples are positioned using a 3-axis precision stage. The output fiber is butt-coupled on the waveguide output facets using a 5-axis precision stage (Elliott Scientific) and connected to a photodiode power sensor (S151C, Thorlabs). The measured propagation losses of the different core materials are given in Table 2, an example of a hot embossed waveguide array is shown in Figure 6 (left).

Table 2. Measured propagation losses of the fabricated waveguides for different materials and wavelengths.

\begin{tabular}{|c|c|c|}
\hline $\begin{array}{c}\text { Waveguide core } \\
\text { material }\end{array}$ & $\begin{array}{c}\text { Propagation loss @633nm } \\
{[\mathbf{d B} / \mathbf{c m}]}\end{array}$ & $\begin{array}{c}\text { Propagation loss @850nm } \\
{[\mathbf{d B} / \mathbf{c m}]}\end{array}$ \\
\hline OG142 & 2.56 & 1.05 \\
\hline OG198-54 & 0.97 & 0.31 \\
\hline NOA68 & 0.74 & 0.81 \\
\hline
\end{tabular}

In general, we obtain relatively uniform refractive index distributions across the waveguide cross sections and propagation losses which are sufficiently low for the structures to be used as sensor elements, see Figure 6 (right). The 
lowest loss of $0.09 \mathrm{~dB} / \mathrm{cm}$ is achieved using the printing ink Supraflex as core material (measured at a wavelength of 850 $\mathrm{nm}$ [17]). Also, beam splitters with various splitting ratios, low losses and an even distribution of the light intensity over all beam splitter output channels are achieved [17]. Using the same technology, work on the production of single mode waveguides is currently under way.
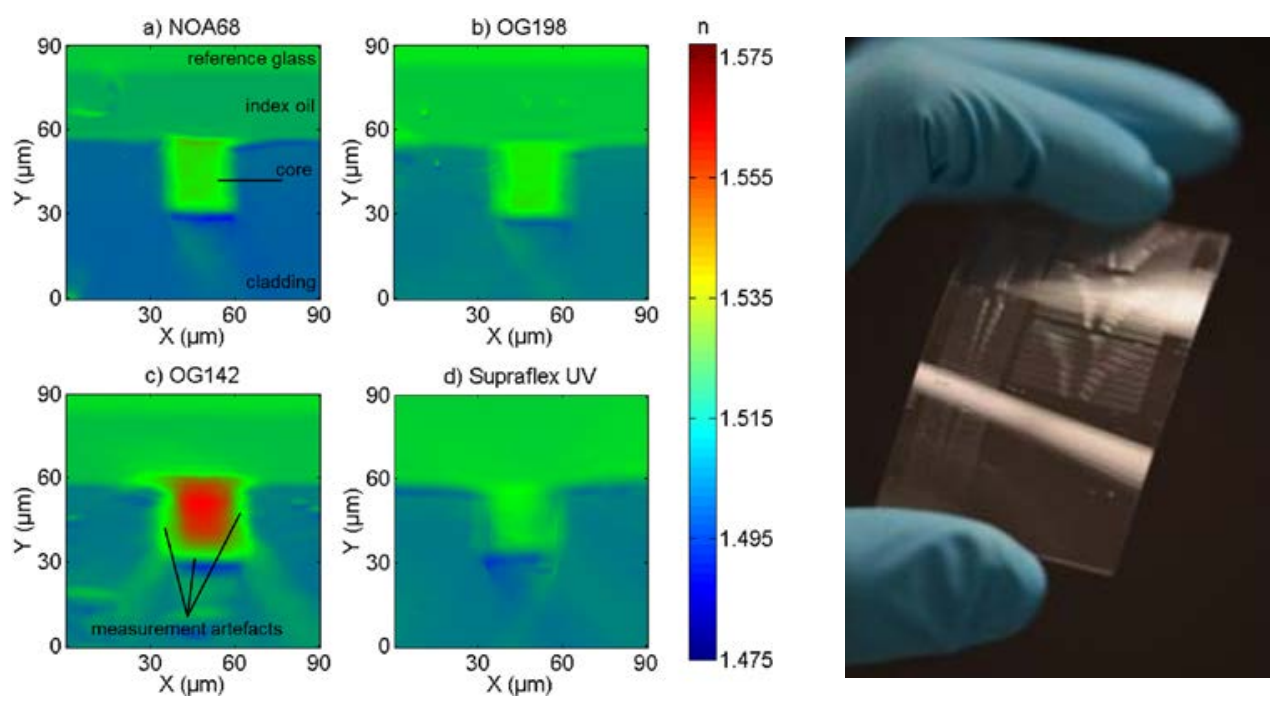

Figure 6. Left: Refractive index distribution for different core materials as measured using a refractive index profiler. Right: Hot embossed flexible waveguide structure array on a thin polymer foil.

\section{b) Structures fabricated by Maskless Lithography}

Figure 7 (left and middle) shows microscope images of a linear waveguide array based on Ormocomp and an array of Ysplitters and Mach-Zehnder interferometer structures of the same material deposited on a fused silica cover slide, respectively. All structures are obtained using an Ormocomp-to-Ormothin ratio of 1:1. The enlargement in Figure 7 (right) depicts in more detail the area indicated by the red box in the middle panel revealing a residual surface roughness which needs to be eliminated to ultimately achieve low-loss polymer-optical elements in the next step. The surface roughness is caused by the relatively large pixel size of the DMD utilized. The results shown in Figure 7 demonstrate that our process is capable of producing simple waveguide structures and also more complex beam splitters and interferometers.
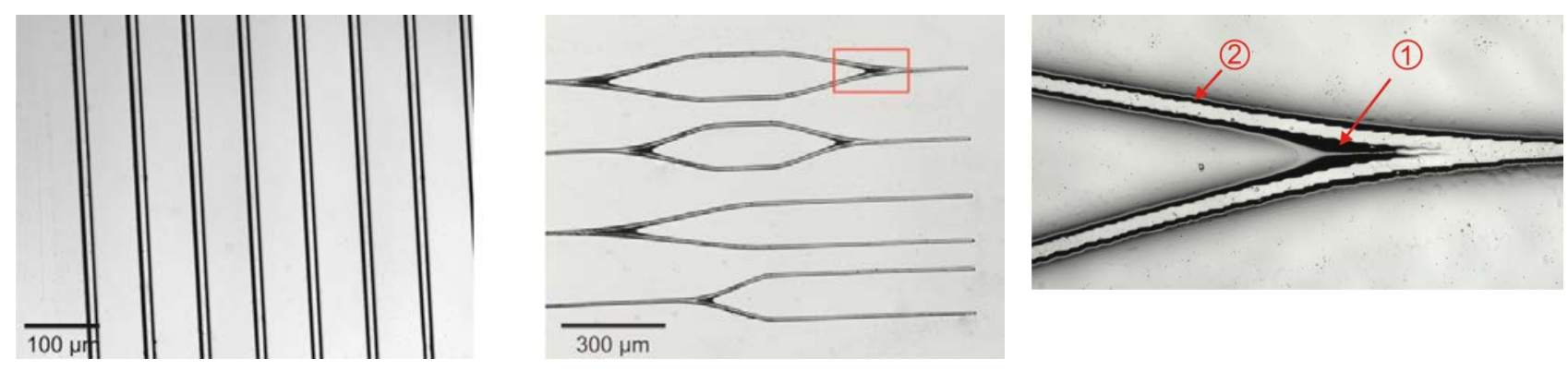

Figure 7. Microscope images of Ormocomp structures fabricated by maskless lithography: linear waveguide array (left) as well as Y-splitters and Mach-Zehnder interferometers (middle). Right: enlargement of the area indicated by the red box in the middle panel (from [16]). 


\section{MANUFACTURED COUPLING STRUCTURES}

To realize polymer-optical sensor networks, a components toolbox containing the basic building blocks is ultimately required. Besides optical waveguides, low loss optical coupling structures and interconnects are necessary. Their purpose is to efficiently couple light in and out of the waveguide array. Depending on the light sources, detectors and sensor architectures utilized, different types of coupling structures come into question, e.g., optical interconnects, micro-mirror couplers and grating couplers.

Self-written waveguides (SWWs) are a promising solution for optical interconnects between very different components such as waveguides, fibers or light sources and detectors. They feature low optical losses and can be realized at low cost. The most promising approach consists of using two lights fields for their realization, as illustrated in Figure 8 (left), see also [20] for details. In the first step, 5-axis precision stages (Elliott Scientific) were used to butt-couple two optical fibers, for example. An UV curable monomer was then deposited in the gap between both fibers (yellow region in Figure 8). After deliberate separation of the fibers to a pre-defined variable distance, laser light at wavelengths of $638 \mathrm{~nm}$ and $780 \mathrm{~nm}$ was coupled into the fibers from opposite sides. In the overlap region of the light fields in between the fibers, i.e. where the curable monomer is applied, polymerization occurs. As a consequence, an optical interconnect forms due to two-wavelength absorption. Compared to the previous approach developed in our group this technique does not require UV laser sources for writing of the waveguides and is also suited to create bent optical interconnects, see [3, 20] for details. Also, the writing process can be controlled externally by an additional third light field which is very convenient and easy to implement. After the writing process the cladding is cured through UV flood exposure. An example of such a fabricated interconnect is shown in Figure 8 (right). The measured propagation loss of the self-written waveguides is about $0.8 \mathrm{~dB} / \mathrm{cm}$ which is acceptable for sensor applications. As the distance between the two coupled fibers can be very small, low interconnect losses of $0.01 \mathrm{~dB}$ were obtained.

1) Writing process

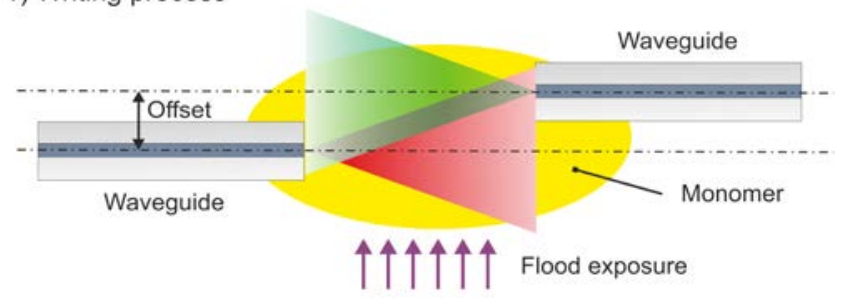

2) Rigid connect in cured polymer
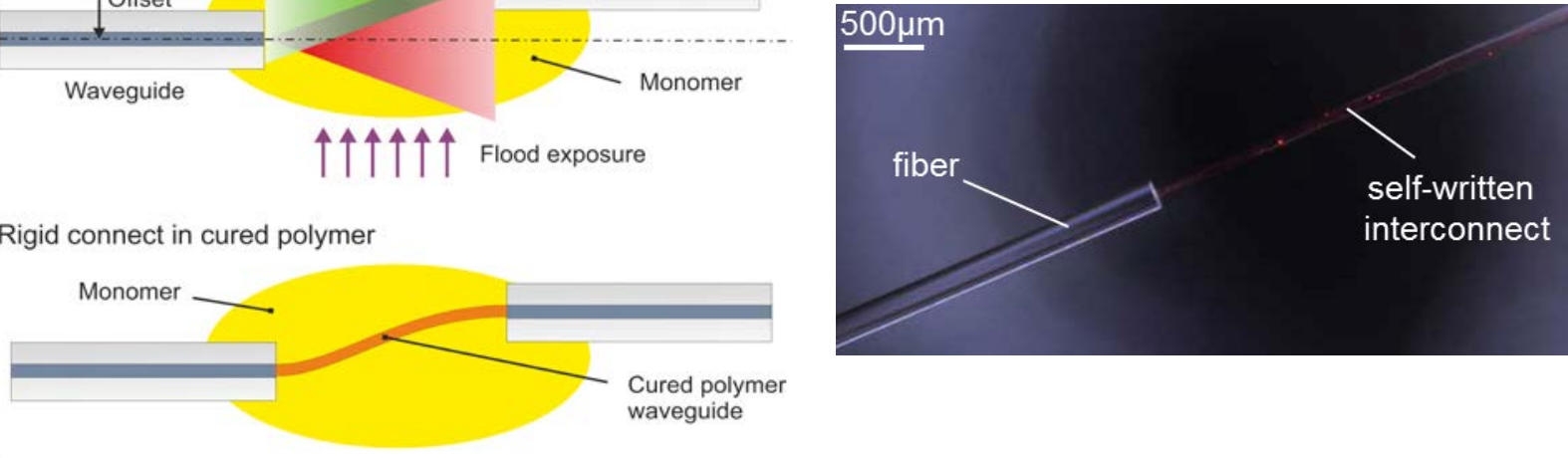

Figure 8. Left: Sketch of the self-written interconnect approach achieved by using two different light fields for the writing process. Right: Microscope image of a fabricated self-written interconnect (from [9]).

The presented optical interconnects are suited for coupling planar waveguides with fibers or side emitting laser diodes, for example. However, for vertical emitting laser diodes (VCSEL) or organic laser diodes and photo detectors which are also employed in our work, light coupling through micro-mirrors or optical gratings is more suited. The case of micromirrors is illustrated in Figure 9. An angled surface is created at the facet of the respective waveguide (Figure 9, top panel a)) using a slotting process. Then, light propagating orthogonal to the waveguides is reflected on the interface between the core and air through total internal reflection and propagates in the waveguide. For the slotting process, a diamond tool was used. Typical results achieved are also shown in Figure 9. 
a)

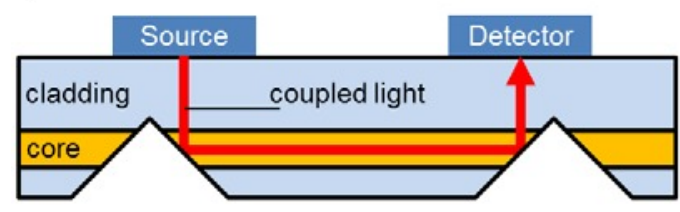

b)

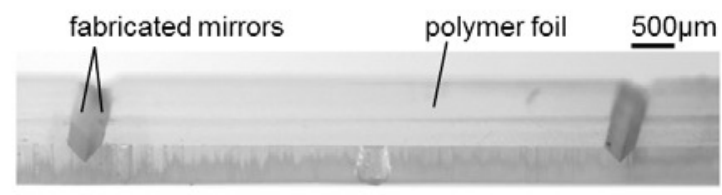

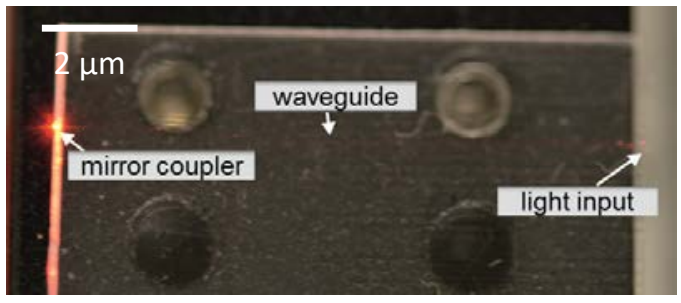

Figure 9. Light coupling through a mirror structure. Top panel: a) Sketch of the concept. b) Result of a slotting fabrication process. Bottom panel: Photograph of manufactured waveguide equipped with a micro-mirror for outcoupling of the light

(from [9]). c) image of light being coupled out from polymer waveguide using a mirror coupler.

The grating couplers on the other side are produced on the surface of embedded waveguides. Light incident orthogonal to the surface is diffracted on the grating and coupled into the waveguide. The task is to choose the right grating dimensions (height, period) which optimally couples the light into the waveguide (see Figure 10, top panel a)). In a first attempt, we fabricated grating structures through hot embossing by using a silicon based master tool. The result of this process is shown Figure 10, top panel b). Also, a scanning electron microscope (SEM) image of the fabricated grating structure is shown in the bottom panel. Coupling efficiencies above $50 \%$ were achieved with an additional silver coating of the waveguide surface.

a)

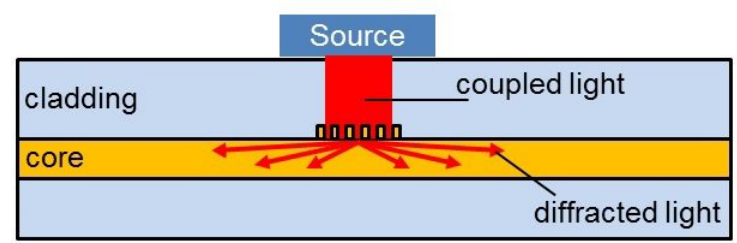

b)

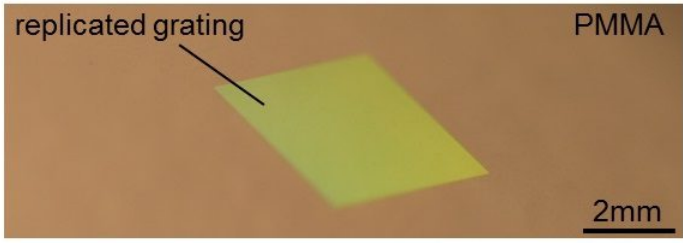

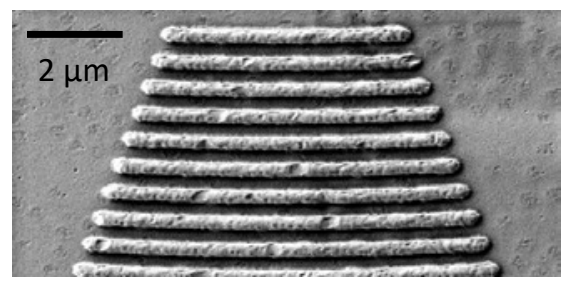

Figure 10. Light coupling through a grating structure. Top panel: a) Sketch of the concept. b) Photography of a hot embossed grating structure. Bottom panel: SEM image of a hot embossed transmission surface grating (from [9]). c) SEM image of hot embossed focusing grating.

By combination of the manufacturing techniques described above, we realized a simple integrated optical transmission line consisting of a semiconductor laser diode, as self-written optical interconnect and a hot embossed polymer-optical waveguide, for example, which will serve as basic building block for more complex sensor networks, see Figure 11 (left). Furthermore, using reactive lamination a structure with embedded polymer optical fibers was created, Figure 11 (right). The approach already presented in [17] employs an active lamination agent to join poly(methyl methacrylate) (PMMA) substrates and a cyclic olefin polymer (COP) monofilament, thus, creating a monolithic, all-polymer optical waveguide. This approach complements the hot embossing technology in or group for the fabrication of polymer-optical components and is, in particular, suited for future production using a roll-to-roll process. 

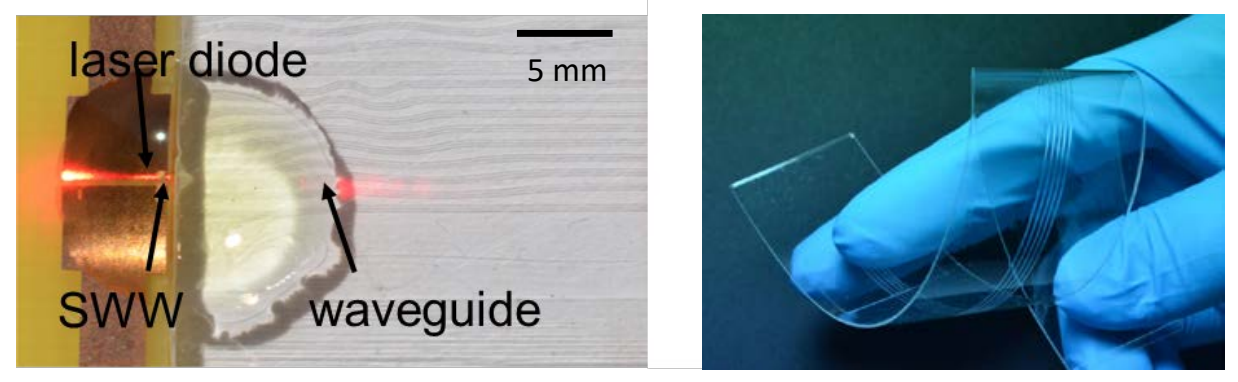

Figure 11. Left: Integrated optical transmission line. Right: Laminated polymer foil structure containing polymer-optical fibers (POFs).

\section{SIMPLE OPTICAL STRAIN, TILT AND HUMIDITY SENSOR DEVICES}

The waveguide and coupling structures developed so far were employed for the realization of simple sensor systems. In the remainder we restrict ourselves to a few illustrative cases and refer to the given literature for a more comprehensive discussion. For example, we demonstrate two concepts for optical strain measurement which rely on suitably arranged waveguide arrays. Figure 12 sketches the concept for strain sensing (left) which can also be used for in-plane tilt sensing (right). The waveguide array consists of five waveguide pairs separated by an elongation zone. The function is based on the analysis of the optical coupling efficiency between both waveguide arrays upon inducing strain or tilt, as indicated in Figure 12 (left). A force applied to the array leads to a change in the configuration of the waveguide pairs and thus to a variation of the transmitted intensity. This variation is different for strain or tilt so that discrimination between the two quantities is possible $[7,8]$. In the first prototypes which were assembled by hand, the sensitivity of the different waveguide pairs varied due to manufacturing inaccuracies, as illustrated in Figure 13 (left). For a damaged or nonoptimally aligned waveguide pair the slope of the intensity curve $I$ as function of elongation $l$ is shallower which corresponds to a lower sensitivity. However, such manufacturing errors can be compensated by averaging as demonstrated in [8] and indicated in Figure 13 (right). The achieved sensitivities for strain $(8.19 \mathrm{mV} / \mu \mathrm{m}$, corresponding to $60 \mathrm{~m} \varepsilon)$ and tilt $(72.04 \mathrm{mV} / \mathrm{mrad})$ are very promising, in particular in view of the large relative elongation of several percent possible with such polymer-based systems. Thus, the device lends itself for monitoring of the structural health of large components, for example.
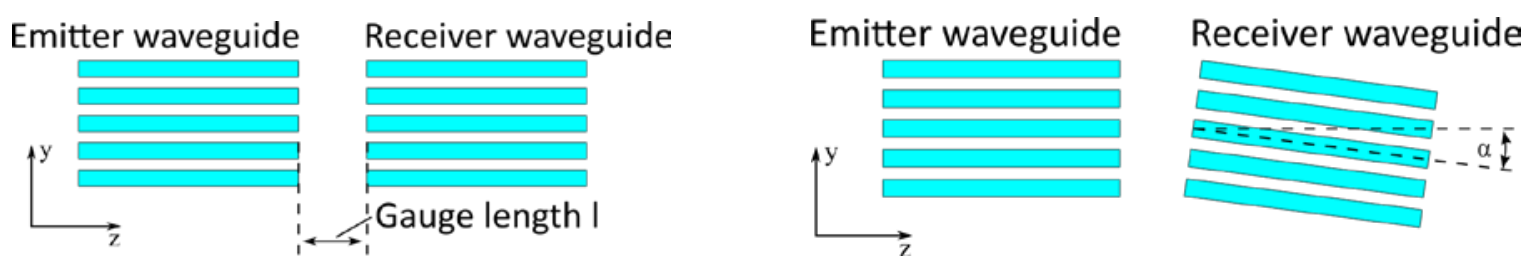

Figure 12. Sketch of the concept of the planar optical strain (left) and tilt sensor (right) based on intensity variation [7].

In a second approach we use diffraction of white light on a diffractive optical element (DOE) inscribed into a polymer substrate by using femtosecond laser radiation [18] for measurement of strain orthogonal to the grating direction. The induced strain can be detected via a spectral change of the diffracted light pattern or, as exemplified in this case, by a change in the spatial distribution of the diffraction pattern of a spectrally narrower light spectrum. Figure 14 (left) shows a schematic of the sensor concept. Its main elements are an emitter waveguide, a diffraction grating (indicated in the left panel, not visible), and a fanout region consisting of five collector waveguides to detect the diffracted light. These elements are separated by free propagation zones. In the middle panel in Figure 14 the realized prototype device is shown.

In brief, after hot embossing of the waveguide structures the diffraction grating was inscribed using femtosecond laser radiation in the direction indicated in Figure 14 (left). Then a force was applied in the direction of the grating and the intensity of the transmitted diffraction pattern evaluated. From the center of gravity of the transmitted light the induced change in the grating period was deduced. In this very preliminary study changes in the grating period on the order of 
$10 \mathrm{~nm}$ could be detected (Figure 14 (right)). However, further systematic studies and improved statistics are required to actually quantify the sensitivity towards strain and the reproducibility and reliability of the devices manufactured. Also, work towards calibration of the device (applies to all sensors shown here) is under way.
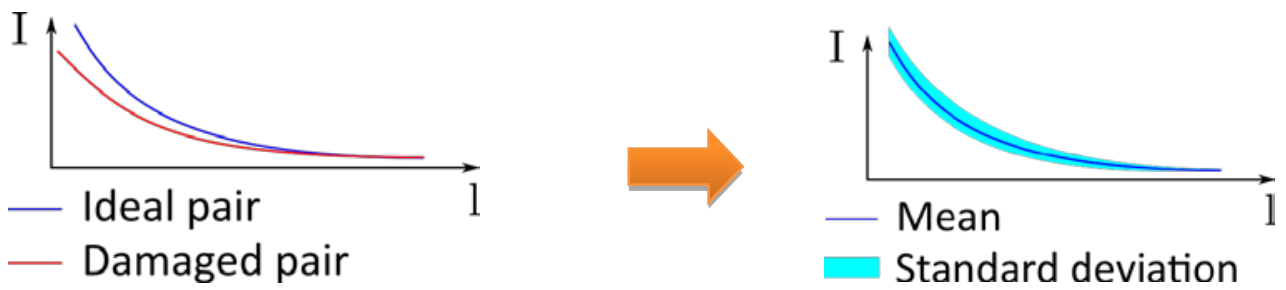

Figure 13. Compensation of manufacturing errors leading to different sensitivity curves (intensity $I$ versus elongation length l) (left) by averaging of several waveguide pairs (right).
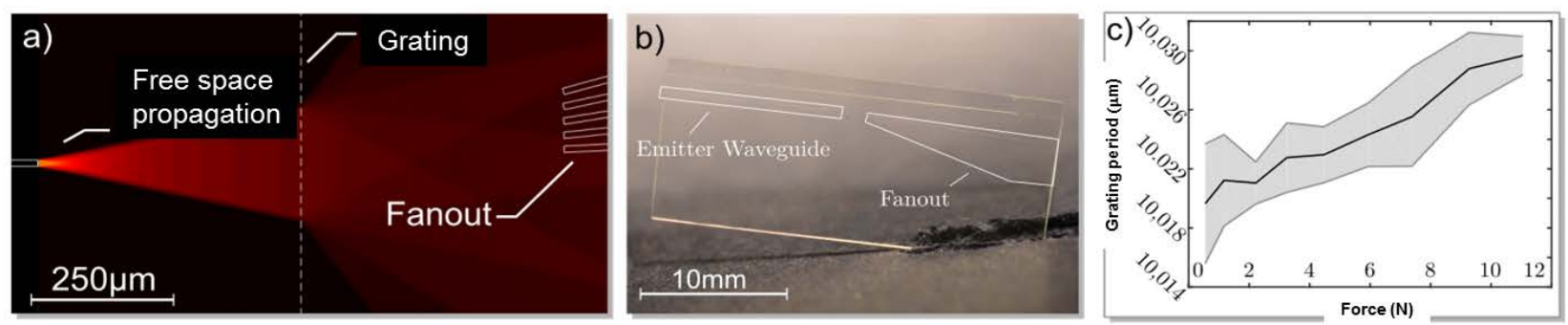

Figure 14. Left: Sketch of chromatic sensor concept. Middle: realized planar polymer structure for the sensor. Right: Change of grating period versus force induced, see also [15]. Grey band corresponds to measurement errors.
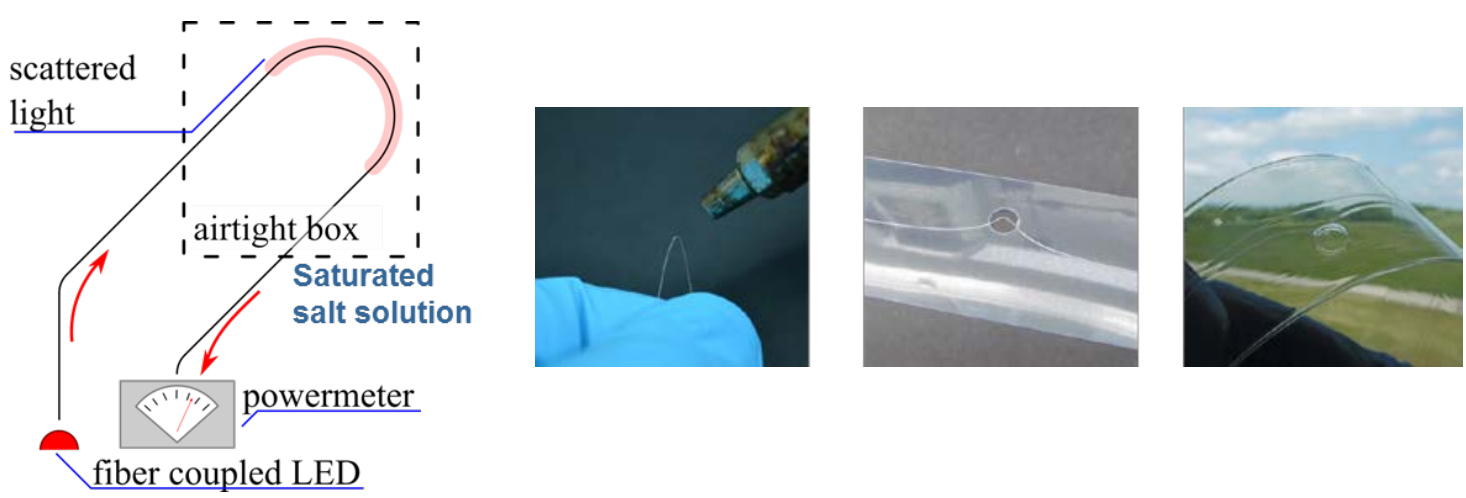

Figure 15. Sketch (left) and photographs (middle to right) of different aspects of the humidity sensor realized using reactive lamination and spray coating of polymer foils and polymer optical fibers [19].

Finally, using reactive lamination we also realize a humidity sensor prototype. It is based on a U-bend polymer-optical waveguide (POF) which is cladded with poly(N,N-dimethylacrylamide) (PDMAA) in the sensing region. The cladding region changes its scattering properties upon absorption of humidity, thus varying the light intensity transmitted through the sensor by virtue of the evanescent field propagating outside the waveguide, see Figure 15 (left). The photographs in Figure 15 (middle to right) show different aspects of the realization of the sensor. The U-bend was manufactured using a polymer-optical fiber and a heat gun (first photograph). Then, reactive lamination was utilized to embed the sensor in between two PMMA substrate foils with an opening left for the sensor area (second photograph). Finally, the photograph on the right in Figure 15 shows that relatively small and flexible humidity sensor devices could be produced by this technique. For the measurement the device placed in an airtight box, incoherent light from a high power LED (Thorlabs, M625F1) is coupled into the sensor and the transmitted power is detected by a power meter together with a fiber coupled 
photodiode detector (Thorlabs, PM200 and S151C, respectively). The environmental humidity outside the box is also measured with a thermo-hygrometer. The sensor is placed in a petri dish with a saturated salt solution, which allows adjusting the relative humidity inside between $11.4 \%$ and $85.1 \%$ at a temperature of $20{ }^{\circ} \mathrm{C}$. Reliable operation and relatively short response times in the range of a few 10 seconds were achieved with the current prototype system.

\section{DISCUSSION AND CONCLUSION}

In this work, we describe manufacturing processes for all-polymer multimode optical waveguide structure, couplers, beam splitters, and diffraction gratings. Polymer-optical waveguides are realized by a combination of hot embossing, doctor blading and UV curing. As substrate material the thermoplastic PMMA is used which serves as waveguide cladding in this case. Several low-cost optical adhesives are employed as core materials. The realized waveguide arrays are characterized by measuring the refractive index and propagation losses, among others. Transmission losses as low as $0.09 \mathrm{db} / \mathrm{cm}$ are achieved which render the structures suitable for optical sensor applications. Furthermore, we manufacture diffraction gratings using the same technique as well as beam splitters and interferometric structures by maskless UV-lithography. We briefly discuss the implementation of the presented processes in a roll-to-roll setup to increase throughput and further reduce costs. Furthermore, we describe different light coupling solutions to integrate micro-optical components, light sources and detectors into planar polymer waveguide arrays. The developed optical interconnects based on a self-writing process reaches coupling losses as low as $0.01 \mathrm{~dB}$. Further, we discuss light coupling by mirror couplers and grating couplers and present first experimental results. Finally, we describe results for simple polymer-optical sensor concepts for strain, tilt, and humidity measurement based on intensity or chromatic variation. Current work focuses on the generation of single mode polymer-optical waveguides, the improvement of the coupling structures and the realization of 2D sensor arrays and networks for distributed sensing. Also, methods for validation and calibration of the sensor systems realized are investigated. Ultimately, we aim at implementation of the technology into roll-to-roll process chains for high-throughput manufacturing. Also, we envision utilizing the sensor systems created for application, e.g., in the life sciences, medicine or environmental analytics, possibly combined with optofluidics or lab-on-chip devices.

\section{ACKNOWLEDGMENTS}

This work was funded by the German Research Foundation (DFG) in the framework of the collaborative research center TRR 123 "PlanOS”.

\section{REFERENCES}

[1] Ye, W. N., Xiong, Y., "Review of silicon photonics: history and recent advances," Journal of Modern Optics 60(16), 1299-1320 (2013)

[2] Tong, X. C. [Advanced Materials for Integrated Optical Waveguides], Springer, Switzerland, (2014)

[3] Günther, A., Petermann, A. B., Gleissner, U., Hanemann, T., Reithmeier, E., Rahlves, M., Wollweber, M., Morgner, U., Roth, B., "Cladded self-written multimode step-index waveguides using a one-polymer approach," Optics Letters 40(8), 1830-1833 (2015)

[4] J. Guo, M. Niu, C. Yang, "Highly flexible and stretchable optical strain sensing for human motion detection," Optica, Volume 4, Issue 10, 1285-1288 (2017)

[5] Rezem, M., Günther, A., Rahlves, M., Roth, B. and Reithmeier, E., "Hot Embossing of Polymer Optical Waveguides for Sensing Applications," Procedia Technology 15, 514-520 (2014)

[6] Rezem, M., Günther, A., Petermann, A. B., Kelb, C., Rahlves, M., Wollweber, M., Roth, B., "Production and characterization of all-polymer based optical waveguides and interconnects," Proc. DGAO 116, (2015)

[7] Kelb, C., Reithmeier, E., Roth, B., "Planar integrated polymer-based optical strain sensor," Proc. SPIE 8977, 89770Y (2014)

[8] Kelb, C., Rahlves, M., Reithmeier, E., Roth, B., "Realization and Performance of an All-Polymer Optical Planar Deformation Sensor," IEEE Sensors Journal 15(12), $7029-7035$ (2015) 
[9] Rezem, M., Kelb, C., Günther, A.,Rahlves, M., Reithmeier, E., Roth, B., "Low-cost fabrication of optical waveguides, interconnects and sensing structures on all-polymer-based thin foils," Proceedings Volume 9751, Smart Photonic and Optoelectronic Integrated Circuits XVIII; 975112 (2016)

[10] Worgull, M. [Hot Embossing: Theory and technology of microreplication], William Andrew, United Kingdom, (2009)

[11] Lan, S., Lee, H. J., Lee, S. H., Ni, J., Lai, X., Lee, H. W., Song, J. H., Lee, M. G., "Experimental and numerical study on the viscoelastic property of polycarbonate near glass transition temperature for micro thermal imprint process," Materials \& Design 30(9), 3879-3884 (2009)

[12] Rezem, M., Günther, A, Rahlves, M., Roth, B., Reithmeier, E., "Fabrication and Sensing Applications of Multilayer Polymer Optical Waveguides", Procedia Technology 6, 517-523 (2016)

[13] Akin, M., Rezem, M., Rahlves, M., Cromwell, K., Roth, B., Reithmeier, E., Wurz, M.M., Rissing, L., Maier, H.-J., "Direct hot embossing of micro-elements by means of photostructurable polyimide," Journal of Micro/Nanolithography MEMS MOEMS 15(3), 034506 (2016)

[14] Schlangen, S., Ihme, M., Rahlves, M., Roth, B., "Autofocusing system for spatial light modulator-based maskless lithography," Applied Optics 55(8), 1863-1870 (2016)

[15] Rahlves, M., Kelb, C., Rezem, M., Schlangen, S., Boroz, K., Gödecke, D., Ihme, M., Roth, B., "Digital mirror devices and liquid crystal displays in maskless lithography for fabrication of polymer-based holographic structures," Journal of Micro/Nanolithography MEMS MOEMS 14(4), 041302 (2015)

[16] Rahlves, M., " Fabrication of polymer based integrated photonic devices by maskless lithography," Proceedings of SPIE Vol. 9745, 97450D (2016)

[17] Rezem, M., Günther, A., Roth, B., Reithmeier, E., Rahlves, M., "Low-Cost Fabrication of All-Polymer Components for Integrated Photonics," Journal of Lightwave Technology 35(2), 299-308 (2017)

[18] Kelb, C., Pätzold, W., Morgner, U., Rahlves, M., Reithmeier, E., Roth, B., "Characterization of femtosecond laser written gratings in PMMA using a phase-retrieval approach", Optical Materials Express 6(10), 3202-3209 (2016)

[19] Kelb, C., Körner, M., Prucker, O., Rühe, J., Reithmeier, E., Roth, B., "A planar low-cost full-polymer optical humidity sensor," Procedia Technology 6, 530-536 (2016)

[20] Günther, A., Schneider, S., Rezem, M., Wang, Y., Gleissner, U., Hanemann, T., Overmeyer, L., Reithmeier, E., Rahlves, M., Roth, B., "Automated misalignment compensating interconnects based on self-written waveguides," Journal of Lightwave Technology 35(13), 2678 - 2684 (2017) 